\title{
Ecclesia disputans
}

Die Konfliktpraxis vormoderner Synoden zwischen Religion und Politik

von Christoph Dartmann, Andreas Pietsch und Sita Steckel

Im I5. Kapitel der Apostelgeschichte schildert der Evangelist Lukas in idealtypischer Weise, wie nach seiner Ansicht ein Konflikt innerhalb der sich formierenden Christenheit beizulegen ist. Er schildert, es habe in Antiochia, dem damaligen Wirkungsort des Apostels Paulus, einen heftigen Konflikt um die Bedingungen gegeben, unter denen Menschen, die bis dahin nicht dem Judentum angehörten, zum Christentum konvertieren können. Christen aus Judäa hätten gefordert, die sogenannten Heidenchristen müssten sich zunächst beschneiden lassen und könnten sich erst dann der christlichen Gemeinde anschließen. Paulus und Barnabas hätten energisch widersprochen, und so habe man in Antiochia beschlossen, eine Delegation nach Jerusalem zu entsenden, um die Apostel und Ältesten der dortigen Gemeinde um eine Klärung zu bitten. In Jerusalem habe es erneut Streit gegeben, weil auch dort die Forderung nach einer Beschneidung aller Konvertiten laut geworden sei. Die Apostel und Ältesten, allen voran Petrus und Jakobus, hätten sich aber in gemeinsamer Beratung mit den Glaubensbrüdern aus Antiochia dann für jüdische Minimalanforderungen und gegen die Beschneidung der Heiden entschieden. ${ }^{\mathrm{I}}$ Dieser in einvernehmlicher Beratung gefällte Beschluss sei dann den Christen in Antiochien in einem Schreiben an alle „Brüder und Schwestern in Antiochia, in Syrien und Kilikien, die zu den Heiden gehören“ (Apg 15,23), mitgeteilt worden, das eine Gesandtschaft aus Jerusalem überbrachte. Zur Begründung hieß es darin: „Denn der Heilige

I Die Heidenchristen hätten lediglich die bereits im Buch Levitikus festgelegten Mindestanforderungen an den Umgang mit Juden zu erfüllen, die Maßregeln für die Verhinderung von Idolatrie und unerwünschten sexuellen Aktivitäten sowie für den Fleischverzehr enthielten. Vgl. zum sogenannten Apostelkonzil einführend Martin Ebner, Von den Anfängen bis zur Mitte des 2.Jahrhunderts, in: Bernd Moeller (Hrsg.), Ökumenische Kirchengeschichte. Bd. I: Von den Anfängen bis zum Mittelalter. Darmstadt 2006, I5-57, hier 3I-35; Thomas Söding, Das Apostelkonzil als Paradebeispiel kirchlicher Konfliktlösung. Anspruch, Wirklichkeit und Wirkung, in: Joachim Wiemeyer (Hrsg.), Dialogprozesse in der katholischen Kirche. Begründungen - Voraussetzungen - Formen. Paderborn u.a. 2013, 25-34. 
Geist und wir haben beschlossen, euch keine weitere Last aufzuerlegen“(Apg I 5,28). Der Brief sei in Antiochia verlesen und dankbar angenommen worden.

Es ist strittig, ob das von Lukas geschilderte ,Apostelkonzil‘ an den Anfang der Tradition christlicher Synoden gestellt werden kann. Dennoch beinhaltet es in nuce Elemente, die für die christliche synodale Tradition prägend werden sollten: Strittige Fragen werden vor ein kollektives Gremium getragen, das aus herausragenden Vertretern der Gemeinden besteht - Lukas nennt Apostel und Älteste, später sollten es vor allem die Bischöfe sein, die in Synoden zusammenkamen. In diesem Gremium werden Konfliktpunkte in einer um Einvernehmen bemühten, also nicht die Gegensätze scharf herausarbeitenden Weise beraten und zu einer von allen Anwesenden mitgetragenen Entscheidung geführt. Besonders an diesem auch aus politischen Kontexten bekannten Vorgehen ist, dass für diese Entscheidung in religiosis explizit Gottes Bestand erfleht wird, dessen Wirken den gefassten Beschlüssen eine besondere Dignität verleiht. Diese Entscheidung wird schriftlich festgehalten und an andere Gemeinden versandt. Sie beansprucht somit Verbindlichkeit über den Kreis der unmittelbar am Konflikt beteiligten Akteure hinaus - hier werden neben Heidenchristen in Antiochia auch ihre Glaubensbrüder in Syrien und Kilikien genannt. Und die zustimmende Aufnahme dieser Beschlüsse in Antiochia sichert ihre Verbindlichkeit - die Geltung beruht auf ihrer Rezeption.

Berücksichtigt man allerdings die parallele Überlieferung zum sogenannten Apostelkonzil, die Paulus in seinem Galaterbrief bietet, zeigt sich, wie stark Lukas das Geschehen stilisiert. Paulus - als Konfliktpartei sicherlich alles andere als ein uninteressierter, neutraler Beobachter - betont seine eigene Initiative, die die Gemeinde in Jerusalem zu einer Klärung gezwungen habe, und bestreitet damit die Kompetenz der dortigen Apostel und Ältesten, über seine Missionspraxis zu richten. Folgerichtig stellt er die Beratungen als Gespräch ,auf Augenhöhe‘ dar, in dem sein Vorgehen uneingeschränkt bestätigt worden sei, ohne irgendwelche Mindestanforderungen zu nennen (Gal 2,I-IO). Somit erscheint der Bericht der Apostelgeschichte in einem anderen Licht. Seine ,Synodalakten` - die Erzählung sowie der inserierte Brief - stilisieren das Geschehen zu einem Muster konsensorientierter Konfliktbeilegung, in das zugleich klare Vorstellungen einer kirchlichen Institutionalisierung eingeschrieben sind. Unabhängig davon, in welchem Maße dieser stilisierte Bericht dem historischen Geschehen entspricht, erlangt er qua Aufnahme in den Kanon des Neuen Testaments langfristig Geltung. Noch in einem anderen Sinne idealisiert der Bericht des Lukas das Konfliktgeschehen, denn er unterschlägt, dass die Beratungen 
in Jerusalem keineswegs das Ende der Auseinandersetzungen um den richtigen Umgang zwischen Juden- und Heidenchristen bedeutet haben. Paulus berichtet jedenfalls, in der Folgezeit sei Petrus bei ihm in Antiochia gewesen und habe auch gemeinsam mit den Heidenchristen gegessen. Als jedoch andere Jerusalemer Christen zu ihnen gestoßen seien, habe sich Petrus von der Mahlgemeinschaft zurückgezogen aus Angst vor den Beschnittenen. Deswegen habe Paulus ihn öffentlich zurechtgewiesen (Gal 2,I I-2I).

Zusammengenommen bietet die Überlieferung zum sogenannten Apostelkonzil somit ein ambivalentes Bild. Auf der einen Seite lässt sie die Erwartung erkennen, innerchristliche Konflikte ließen sich im Vertrauen auf Gottes Wirken durch gemeinsame Beratungen beilegen, deren schriftlich fixierte Ergebnisse zu einem friedlichen Zusammenleben und damit zu einer Stärkung der Kohärenz zwischen verschiedenen christlichen Gruppen beitragen. Auf der anderen Seite zeigt sie, wie fragil der Kompromiss war, wenn nicht alle Akteure an ihm festhielten. Das Bild einer friedlichen, diskursiven und in geregelten Verfahren erzielten Lösung erweist sich als hochgradig stilisiert. Aber gerade in dieser Stilisierung gewann es doch wieder historische Realität, denn es wurde zu dem Ideal, an dem sich spätere Synoden orientieren konnten.

\section{Kirchliche Entscheidungsfindung im Spannungsfeld von Religion und Politik}

Die offensichtliche Spannung zwischen konflikthaften politischen Realitäten und verschiedenen, rechtlich-politisch wie religiös fundierten Idealen konsensualer Entscheidungsfindung blieb im Verlauf des Mittelalters bekanntlich bestehen. Auftretende Überschneidungen zwischen religiöser und politischer Sphäre waren vielfältig. Einerseits vertraten Synoden innerhalb der lateinischen Kirche den Anspruch, göttlich gefällte Entscheidungen lediglich zu verkünden, beziehungsweise boten sich als Orte und Garanten für eine Ermittlung des göttlichen Willens an. Andererseits waren und blieben synodale Treffen in wesentlicher Weise ,politische` Ereignisse. Während die Meinungsverschiedenheiten zwischen verschiedenen Parteien im Fall des Apostelkonzils auf einen persönlichen Gegensatz zurückgeführt werden, traten im Verlauf des Mittelalters immer deutlicher Spannungen zwischen Stellvertretern bestimmter Gruppierungen und Institutionen hervor - zwischen 
Papst und Bischöfen im Falle einiger hoch- und spätmittelalterlicher Synoden, seit der spätantiken Kaiserzeit aber auch zwischen kirchlichen Amtsträgern und weltlichen Herrschern.

Die Verquickung von Entscheidungsmechanismen aus den Bereichen der weltlichen Politik, des Rechts oder schließlich der religiösen Wahrheitsfindung erscheint somit als Quelle fortwährender Dynamiken: Während Verfahren politischer Willensbildung und gelehrter oder gerichtlich-rechtlicher Problemlösung sich prinzipiell als häufige Vorbilder synodaler Entscheidungsfindung erweisen, wurden auch religiöse Logiken ins Feld geführt - nicht zuletzt dann, wenn es galt, dem ungewollten Eingreifen bestimmter (weltlicher) Akteure Schranken zu setzen. Immer wieder wurde daher die Legitimität bestimmter Rahmungen der Konfliktlösung thematisiert. Die Akten der Synode von Toledo 684 bemerkten etwa, dass Gott nicht befohlen habe, ihn zu debattieren, sondern an ihn zu glauben („Non enim se Deus discutere iubet sed credere“). ${ }^{2}$

Synoden wiesen weiterhin zwar einen zunehmend festen, standardisierten liturgischen Rahmen auf. Für die Art und Weise des Umgangs mit Konflikten und die Formen des Entscheidens über Streitsachen wurden in ordines de celebrando concilio und ähnlichen Texten jedoch nie klare Normen festgeschrieben. Lediglich allgemeine Erwartungen wie die Freihaltung der Diskussion von persönlichen Bindungen und Geldgeschenken oder die Verpflichtung auf ein von Gewalt und Tumulten freies Debattieren wurden geäußert. ${ }^{3}$ Die Nutzung ganz verschiedener Konfliktstrategien blieb daher möglich.

In paradoxer Weise erscheint der Rahmen der Synode somit als historisch variabel, steht jedoch - als Ultima Ratio der Konfliktbeilegung innerhalb der Kirche trotzdem für Konstanz. Dies dürfte nicht zuletzt an der besonderen Leistung der schriftlichen Überlieferung synodaler Entscheidungen liegen: Nicht nur die Konfliktlösung, sondern vor allem die Kommunikation eines Ergebnisses erwies sich im Nachhinein zumeist als Bezugspunkt für deren Akzeptanz und Verbindlichkeit - es sei denn, dass eine Veröffentlichung mit Gegendarstellungen zu kämpfen hatte. Insofern erscheinen nicht nur synodale Versammlungen, sondern auch ihre An-

2 Vgl. den Titel des Beitrags von Christoph Dartmann in diesem Band mit Diskussion der Stelle, 6r f.

3 Vgl. hier nur Herbert Schneider, Die Konzilsordines des Früh- und Hochmittelalters. Ordines de celebrando concilio. Hannover I996, und den Beitrag von Christoph Dartmann in diesem Band, 67-69 bei Anm. II und I7-I8. 
schlusskommunikation und gegebenenfalls ihre Vorläufe als Orte der Konfliktlösung. Die Legitimität von Entscheidungen musste nicht nur während, sondern auch schon im Vorfeld und erst recht nach der Synode diskursiv oder symbolisch hergestellt werden. Dazu trugen nicht zuletzt die Kirchenrechtssammlungen bei, die Synodalbeschlüsse in großer Zahl zusammentrugen und ihre Rezeption langfristig gewährleisteten. ${ }^{4}$

\section{Anliegen und Forschungskontexte der Münsteraner Tagung}

Diese Multidimensionalität synodaler Konfliktpraxis von der Antike bis zur Frühen Neuzeit in den Blick zu nehmen war Ausgangspunkt der Tagung, die im vorliegenden Band zur Publikation gebracht wird. Sie ging von der Beobachtung aus, dass die unterschiedlichen Aspekte synodaler Konfliktbearbeitung bislang für verschiedene Epochen unterschiedlich thematisiert worden sind. Die verschiedenen Forschungsperspektiven können zudem noch stärker aufeinander bezogen werden: Es liegen Ansätze zur Erforschung der rechtlich-rituellen Rahmung von Kirchenversammlungen und viele Studien zu spezifischen Abläufen vor, insbesondere zu den großen Konzilien des I5.Jahrhunderts. Angesichts des typischen Aufeinandertreffens kirchlicher und weltlicher Handlungs- und Deutungsstrukturen auf Synoden erscheint es jedoch sinnvoll, synodal-kirchliche Entscheidungen stärker mit weltlichen Praktiken der Konfliktführung und -lösung in Zusammenschau zu bringen. Um die Verknüpfung religiöser und profaner Elemente der Konfliktlösung herauszuarbeiten, muss zudem die Funktion eines synodalen Rahmens genau betrachtet werden. Die liturgisch klar aus einem Konfliktgeschehen herausgehobene Synode konnte ja eine Konfliktlösung anregen, vorbereiten und sogar erarbeiten - oder lediglich bereits gefundene Übereinkünfte publizieren.

Zur systematischen Entwicklung von Zugängen zu diesen Fragen boten sich drei

\footnotetext{
4 Andreas Thier, Dynamische Schriftlichkeit. Zur Normbildung in den vorgratianischen Kanonessammlungen, in: Zeitschrift der Savigny-Stiftung für Rechtsgeschichte, Kanonistische Abteilung 93, 2007, I-33; Christoph H.F. Meyer, Ordnung durch Ordnen. Die Erfassung und Gestaltung des hochmittelalterlichen Kirchenrechts im Spiegel von Texten, Begriffen und Institutionen, in: Bernd Schneidmüller/Stefan Weinfurter (Hrsg.), Ordnungskonfigurationen im hohen Mittelalter. (Vorträge und Forschungen, Bd. 64.) Ostfildern 2006, 303-4II.
} 
etablierte Forschungsfelder an, deren gemeinsame Bezugspunkte in letzter Zeit immer deutlicher geworden sind. Wesentliche Entwicklungslinien und Ansatzpunkte liegen aus der Erforschung von Konzilien und Synoden vor. ${ }^{5}$ Sie sollten mit Ansätzen der bislang vor allem auf weltliche Kontexte fokussierten historischen Konfliktforschung in Zusammenschau gebracht werden. ${ }^{6}$ Als relevantes Bindeglied bot sich die Forschungsfrage nach der Institutionalisierung, Verrechtlichung und Formalisierung von Verfahren der Entscheidung und Beratung an. ${ }^{7}$ Ziel der Tagung war, die be-

5 Vgl. zur Geschichte von Konzilien und Synoden allgemein die Bände der Konziliengeschichte hrsg. v. Walter Brandmüller, Rh. A: Darstellungen, und Rh.B: Untersuchungen, z. B. Wilfried Hartmann, Die Synoden der Karolingerzeit im Frankenreich und in Italien. (Konziliengeschichte, Rh.A.) Paderborn 1989; Georg Gresser, Die Synoden und Konzilien in der Zeit des Reformpapsttums in Deutschland und Italien von Leo IX. bis Calixt II., I049-I I 23. (Konziliengeschichte, Rh.A.) Paderborn 2006, sowie exemplarisch Franz-Josef Schmale, Systematisches zu den Konzilien des Reformpapsttums im I2.Jahrhundert, in: Annuarium Historiae Conciliorum 6, I974, 2 I-39; Anne J. Duggan, Conciliar Law, I I23-I2 I5. The Legislation of the Four Lateran Councils, in: Wilfried Hartmann/Kenneth Pennington (Eds.), The History of Medieval Canon Law in the Classical Period, I I 40-I 234. Washington, D. C. 2008, 318-366; Nathalie Kruppa/Leszek Zygner (Hrsg.), Partikularsynoden im späten Mittelalter. (Veröffentlichungen des Max-Planck-Instituts für Geschichte/ Studien Zur Germania Sacra, Bd. 2 I g/20.) Göttingen 2006); stellvertretend für die nachreformatorisch-protestantische Tradition Otto Scheib, Die innerchristlichen Religionsgespräche im Abendland. Regionale Verbreitung, institutionelle Gestalt, theologische Themen, kirchenpolitische Funktion mit besonderer Berücksichtigung des konfessionellen Zeitalters (I517-1689). 3 Bde. Wiesbaden 2009; Aza Goudriaan/Fred van Lieburg (Eds.), Revisiting the Synod of Dordt (I6I8-I6I9). Leiden $201 \mathrm{I}$.

6 Vgl. zur Konfliktforschung allgemein Thorsten Bonacker (Hrsg.), Sozialwissenschaftliche Konflikttheorien. Eine Einführung. 4. Aufl. Wiesbaden 2008; Simon Roberts/John L. Comaroff, Rules and Processes. The Cultural Logic of Dispute in an African Context. Chicago/London I981; Simon Roberts, Order and Dispute. An Introduction to Legal Anthropology. Harmondsworth I979. Aus mediävistischer Perspektive (jeweils mit weiteren Verweisen) Wendy Davies/Paul Fouracre (Eds.), The Settlement of Disputes in Early Medieval Europe. Cambridge I992; Gerd Althoff, Spielregeln der Politik im Mittelalter. Kommunikation in Frieden und Fehde. Darmstadt 1997; Knut Görich, Die Ehre Friedrich Barbarossas. Kommunikation, Konflikt und politisches Handeln im I2.Jahrhundert. Darmstadt 200I; Warren Brown/Piotr Górecki (Eds.), Conflict in Medieval Europe. Changing Perspectives on Society and Culture. London 2003; Stefan Esders (Hrsg.), Rechtsverständnis und Konfliktbewältigung. Gerichtliche und außergerichtliche Strategien im Mittelalter. Köln/Weimar/Wien 2007.

7 Vgl. Barbara Stollberg-Rilinger (Hrsg.), Vormoderne politische Verfahren. (Zeitschrift für historische Forschung, Beih. 25.) Berlin 200I; bes. Michael Sikora, Der Sinn des Verfahrens. Soziologische Deutungsangebote, in: ebd. 25-5I; Barbara Stollberg-Rilinger/André Krischer (Hrsg.), Herstellung und Darstellung von Entscheidungen. Verfahren, Verwalten und Verhandeln in der Vormoderne. (Zeitschrift für historische Forschung, Beih. 44.) Berlin 2010. Vgl. weiterhin Hendrik Vollmer, Akzeptanzbeschaffung. Verfahren und Verhandlungen, in: Zeitschrift für Soziologie 25, I996, I47-I64; Matthias Köhler, Strategie und Symbolik. Verhandeln auf dem Kongress von Nimwegen. (Externa-Geschichte der Außenbeziehungen in neuen Perspektiven, Bd. 3.) Köln/Weimar/Wien 20r r. Vgl. für den soziologischen Hintergrund dieser Forschungs- 
reits vorhandenen Berührungspunkte zwischen diesen Feldern genauer auszuloten und weiterzuentwickeln. ${ }^{8}$ Daher wurden nicht die großen Konzilien des Spätmittelalters in den Mittelpunkt gestellt, sondern exemplarische Überlegungen zu großen wie kleinen Synoden und Kirchenversammlungen zwischen Antike und Früher Neuzeit versammelt und in Vorträgen und Kommentaren diskutiert. ${ }^{9}$

Die Überlegungen des vorliegenden Bandes schließen somit an Ergebnisse und methodische Überlegungen unterschiedlicher Forschungstraditionen an. Die Konzilienforschung hat mit ihrer Frage nach dem Selbstverständnis und den Befugnissen synodaler Versammlungen ${ }^{\mathrm{IO}}$ zentrale Grundbeobachtungen vorgelegt: Herr-

richtung Niklas Luhmann, Legitimation durch Verfahren. Frankfurt am Main I 983; Uwe Schimank, Die Entscheidungsgesellschaft. Komplexität und Rationalität der Moderne. Berlin 2005.

8 Vgl. bereits die Verknüpfungen verschiedener Perspektiven in Arbeiten zur rituellen Gestaltung von Kirchenversammlungen, z. B. Johannes Helmrath, Rangstreite auf Generalkonzilien des I5.Jahrhunderts als Verfahren, in: Stollberg-Rilinger (Hrsg.), Vormoderne politische Verfahren (wie Anm.7), I39-I73, sowie schon Hermann Heimpel, Sitzordnung und Rangstreit auf dem Basler Konzil. Skizze eines Themas, in: Johannes Helmrath/Heribert Müller (Hrsg.), Studien zum I 5. Jahrhundert. Festschrift für Erich Meuthen. Bd. I. München I994, I-9; Klaus Schreiner, Wahl, Amtsantritt und Amtsenthebung von Bischöfen. Rituelle Handlungsmuster, rechtlich normierte Verfahren, traditionsgestützte Gewohnheiten, in: Stollberg-Rilinger (Hrsg.), Vormoderne politische Verfahren (wie Anm. 7), 73-I I 8. Von verschiedenen Seiten sind synodale Versammlungen auch mit weltlichen politischen Versammlungen in Zusammenhang gebracht worden, vgl. etwa Jörg Peltzer/Gerald Schwedler/Paul Töbelmann(Hrsg.), Politische Versammlungen und ihre Rituale. Repräsentationsformen und Entscheidungsprozesse des Reichs und der Kirche im Späten Mittelalter. Ostfildern 2009, bes. Jürgen Miethke, Formen der Repräsentation auf Konzilien des Mittelalters, in: ebd. 2 I-36; Jürgen Dendorfer, Inszenierung von Entscheidungsfindung auf den Konzilien des I 5. Jahrhunderts. Zum Zeremoniell der Sessio generalis auf dem Basler Konzil, in: ebd. 37-54. Relevant weiterhin einige Beiträgen des nach der Tagung erschienenen Bandes Bernward Schmidt/Hubert Wolf(Hrsg.), Ekklesiologische Alternativen? Monarchischer Papat und Formen kollegialer Kirchenleitung (I5.-20.Jahrhundert). (Symbolische Kommunikation und gesellschaftliche Wertesysteme, Bd. 42.) Münster 20I3, sowie Leidulf Melve, Assembly Politics and the „Rules-of-the-Game“ (c. 650-I I 50), in: Viator 4 I/2, 2010, 69-90, der Synoden und weltliche Versammlungen ohne Unterscheidungen diskutiert.

9 Nicht nur den Beiträgern, sondern auch den Kommentatoren der Tagung-Gerd Althoff, Claudia Märtl, Steffen Patzold, Barbara Stollberg-Rilinger und Nicola Stricker - sei nochmals herzlich gedankt. Die Kommentatoren Constant J. Mews (der Clare Monagle als Co-Autorin gewann) und Melanie Brunner konnten dankenswerterweise für eigene Beiträge gewonnen werden.

Io $\mathrm{Zu}$ Theorie und Vorstellungen von Konzilien als Leitungsorganen der Kirche vgl. Brian Tierney, Foundations of the Conciliar Theory. The Contribution of the Medieval Canonists from Gratian to the Great Schism. (Studies in the History of Christian Thought, Vol.8I.) 2. Auflage Leiden/New York/Köln I 998 (erste Auflage I955); Bernard Botte/Hilaire Marot/Pierre-Thomas Camelot (Eds.), Le Concile et les conciles. Contribution à l'histoire de la vie conciliaire de l'Église. Paris I96o; Anthony J. Black, Monarchy and Community. Political Ideas in the Later Conciliar Controversy. (Cambridge Studies in Medieval Life and Thought, $3{ }^{\text {rd }}$ 
scher und Päpste, Bischöfe und lokale Eliten, Juristen und Theologen entwickelten eigene Vorstellungen und Überzeugungen zur Beschaffenheit, Rolle und Befugnis von Konzilien - nicht zuletzt anlässlich von Meinungsverschiedenheiten und Konflikten, die auf ihnen zu verhandeln waren. Der Ausgang von Konflikten war insofern oft genug vom eingeschlagenenen Weg der Konfliktlösung mitbestimmt, und wie sich im Spätmittelalter immer deutlicher abzeichnet, verknüpften sich meist klare politische Interessen und Kalküle mit religiösen Überzeugungen zur richtigen Entscheidungsfindung in der Kirche. Es zeigen sich somit episodenhafte, von situativen Konstellationen geprägte Veränderungen des praktischen und theoretischen Verständnisses von Konfliktlösung auf Synoden - wobei Brüche auftreten, wenn eine beteiligte Partei oder Allianz ihre Strategie der Konfliktbeeinflussung durchzusetzen verstand, wie dies mit der Kirchenreform des Hochmittelalters, während der Reformkonzilien oder wieder mit dem Bruch der Reformation geschah.

Die spätmittelalterliche Bewegung des Konziliarismus und die konkreten Abläufe der großen Konzilien des I 5. Jahrhunderts wie Pisa, Konstanz und Basel ${ }^{\text {II }}$, die be-

Ser., Vol. 2.) Cambridge I970; ders., Council and Commune. The Conciliar Movement and the FifteenthCentury Heritage. London I979; Hermann Josef Sieben, Die Konzilsidee der Alten Kirche. (Konziliengeschichte, Rh. B.) Paderborn/München/Wien/Zürich I979; ders., Die Konzilsidee des Lateinischen Mittelalters 847-I378. (Konziliengeschichte, Rh.B.) Paderborn/München/Wien/Zürich I984; ders., Die Konzilsidee von der Reformation bis zur Aufklärung. (Konziliengeschichte, Rh.B.) Paderborn/München/Wien/ Zürich I988; Hans-Jürgen Becker, Die Appellation vom Papst an ein allgemeines Konzil. Historische Entwicklung und kanonistische Diskussion im Späten Mittelalter und in der Frühen Neuzeit. (Forschungen zur kirchlichen Rechtsgeschichte und zum Kirchenrecht, Bd. I7.) Köln/Wien I988; Constantin Fasolt, Council and Hierarchy. The Political Thought of William Durant the Younger. (Cambridge Studies in Medieval Life and Thought, $4^{\text {th }}$ Ser., Vol. I6.) Cambridge/New York I99I; Francis Oakley, The Conciliarist Tradition. Constitutionalism in the Catholic Church, I300-1870. Oxford 2003; Bernward Schmidt, Die Konzilien und der Papst. Von Pisa (I409) bis zum Zweiten Vatikanischen Konzil (I962-65). Freiburg im Breisgau 2013.

I I Vgl. zu den Konzilien des I5.Jahrhunderts Heribert Müller, Die kirchliche Krise des Spätmittelalters: Schisma, Konziliarismus und Konzilien. (Enzyklopädie deutscher Geschichte, Bd. 90.) München 20I 2; Heribert Müller/Johannes Helmrath, Die Konzilien von Pisa (I409), Konstanz (I4I4-I4I8) und Basel (I43II449). Institution und Personen. (Vorträge und Forschungen, Bd. 67.) Ostfildern 2007, bes. Helmrath/ Müller, Zur Einführung, ebd.9-30; Ansgar Frenken, Die Erforschung des Konstanzer Konzils (I4I4-I4I 8) in den letzten Ioo Jahren. Paderborn I 995 (= Annuarium Historiae Conciliorum 25/I-2, I 993); Francis Oakley, Verius est licet difficilius. Tiemey's Foundations of the Conciliar Theory after Forty Years, in: Studies in the History of Christian Thought 7 I, I 996, I 5-34; Johannes Helmrath, Das Basler Konzil I 43 I-I 449. Forschungsstand und Probleme. (Kölner Historische Abhandlungen, Bd. 32.) Köln/Wien I987; Thomas Prügl, Die Ekklesiologie Heinrich Kalteisens OP in der Auseinandersetzung mit dem Basler Konziliarismus. Mit einem Textanhang. (Veröffentlichungen des Grabmann-Institutes zur Erforschung der mittelalterlichen Theologie und Philosophie, NF., Bd. 40.) Paderborn I 995; Gerald Christianson/Thomas M. Izbicki/Christopher M. Bel- 
sonders im Umfeld des II. Vatikanischen Konzils stärkeres Interesse auf sich zogen $^{\mathrm{I} 2}$, dürfen heute als einer der am besten erforschten Abschnitte solcher Transformationen gelten. Doch haben sich in der Konzilsforschung auch jenseits der großen Themen von Superiorität und Ekklesiologie Fragen entwickelt, die sich auf die aufscheinenden Konvergenzen der vorliegenden Beiträge beziehen lassen. Die Erforschung der Verfahrensweisen - und damit auch der Konfliktlösung - der teils zur „Behörde“ ausgebauten spätmittelalterlichen Konzilien ist weit vorangeschritten. ${ }^{\text {I3 }}$ Doch bliebe einiges für die vielgestaltigen und immer wieder Veränderungen unterworfenen Formen der Entscheidungsfindung und Konfliktlösung auf sonstigen großen und kleinen Synoden zu tun. Zudem ist immer wieder gefragt worden, welche Rolle Medien und Öffentlichkeit für die großen Konzilien spielten. Setzte man etwa auf die Kraft argumentativer Klärung und trat in (zumindest scheinbar) ergebnisoffene Verhandlungen ein, auch wenn das zu tagelangen, aber kaum persuasiv wirk-

litto (Eds.), The Church, the Councils, and Reform. The Legacy of the Fifteenth Century. Washington, D. C. 2008; Jürgen Dendorfer/Claudia Märtl (Hrsg.), Nach dem Basler Konzil. Die Neuordnung der Kirche zwischen Konziliarismus und monarchischem Papat (ca. I450-I475). (Pluralisierung \& Autorität, Bd. I3.) Münster 2008; Heribert Müller (Hrsg.), Das Ende des konziliaren Zeitalters (I440-I450). Versuch einer Bilanz. (Schriften des Historischen Kollegs, Kolloquien, Bd. 86.) München 20I 2; Ulrich Horst, Juan de Torquemada und Thomas de Vio Cajetan. Zwei Protagonisten der päpstlichen Gewaltenfülle. (Quellen und Forschungen zur Geschichte des Dominikanerordens, NF., Bd. I9.) Berlin 20 I 2; Jan Keupp/Jörg Schwarz, Konstanz I4I4-I4I 8. Eine Stadt und ihr Konzil. Darmstadt 2013.

I2 Vgl. zusammenfassend Heribert Müller, Konzilien des I5.Jahrhunderts und Zweites Vatikanisches Konzil. Historiker und Theologen als Wissenschaftler und Zeitgenossen, in: Dieter Hein/Klaus Hildebrand/Andreas Schulz (Hrsg.), Historie und Leben. Der Historiker als Wissenschaftler und Zeitgenosse. Festschrift für Lothar Gall. München 2006, I I5-I35.

I3 Der Begriff der Behörde u.a. bei Johannes Helmrath, Das Konzil als Behörde. Eine unbekannte Kanzleiordnung des Basler Konzils von I439, in: Michael Matheus/Andreas Rehberg (Hrsg.), Kurie und Region. Festschrift für Brigide Schwarz zum 65. Geburtstag. (Geschichtliche Landeskunde, Bd. 59.) Stuttgart 2005, 93-I I 2. Vgl. mit verschiedenen Aspekten zur Konfliktführung bes. Thomas Graumann, Die Kirche der Väter. Vätertheologie und Väterbeweis in den Kirchen des Ostens bis zum Konzil von Ephesus (43I). (Beiträge zur historischen Theologie, Bd. II8.) Tübingen 2002; Ramsay MacMullen, Voting About God in Early Church Councils. New Haven, CT/London 2006; Gresser, Die Synoden (wie Anm. 4), 523-564; Hartmann, Synoden (wie Anm.4), I-Io; Wolter, Die Synoden (wie Anm.4), 455-478; Raymonde Foreville, Procédure et débats dans les conciles médievaux du Latran (I I23-I2 I5), in: dies., Gouvernement et vie de l'Église au Moyen-Âge. Recueil d'études. (Variorum Collected Studies Series, Vol.95.) London I979, 2 I-37; Johannes Laudage, Ritual und Recht auf den päpstlichen Reformkonzilien, in: Annuarium Historiae Conciliorum 29, I 997, 287-334; stellvertretend für mehrere Arbeiten zum I 5.Jahrhundert Helmrath, Das Basler Konzil (wie Anm. I I), sowie Stefan Sudmann, Das Basler Konzil. Synodale Praxis zwischen Routine und Revolution, Tradition, Reform, Innovation (Studien zur Modernität des Mittelalters, Bd. 8.) Frankfurt am Main/New York 2005; vgl. auch zahlreiche der in Anm 5. aufgeführten neueren Beiträge. 
samen Debatten führte, wie Thomas Prügl auf der Münsteraner Tagung am Beispiel der Disputation mit den hussitischen Theologen auf dem Basler Konzil diskutierte? ${ }^{\text {I4 }}$ Oder sind gerade die überlieferten Traktate der Reformkonzilien des I5. Jahrhunderts eigentlich als Stellungnahmen und Argumentsammlungen zu deuten, was im Gegenzug dem Kontext der Versammlung einen eher zeremoniell rahmenden, freilich dennoch wichtigen, etwa zeitlich strukturierenden Charakter ${ }^{15}$ zuwei- $^{2}$ sen würde? Zudem ist vielfach beschrieben worden, dass die großen Konzilien als „Foren der öffentlichen Meinung“ und zudem als Drehscheiben intellektueller Einflüsse fungierten. ${ }^{16}$ Doch wie betteten sich verschiedene Mechanismen der synodalen Entscheidungsfindung in vormoderne Formen von Öffentlichkeit ein? Schon an den großen Konzilien des I5.Jahrhunderts wird sehr deutlich, dass synodale Verhandlungen in komplexe Zusammenhänge eingebunden waren, die keineswegs sämtlich unter ähnlichen Bedingungen abliefen, sondern eine Vielzahl von unterschiedlichen Situationen der Vor- und Nachbereitung, des formellen und informellen Verhandelns, öffentlichen oder begrenzt öffentlichen Argumentierens einschlossen. Eine vergleichbare Komplexität dürfte sich für weitere Synoden des Untersuchungszeitraums postulieren lassen.

An dieser Stelle zeigen sich daher zahlreiche mögliche Querverbindungen zur Konfliktforschung. Der seit Längerem in die historische Forschung eingeführte Begriff des ,Konflikts‘ bietet für die Erforschung historischer Formen des Politischen grundsätzlich die Möglichkeit, nach Mechanismen der Konfliktführung und -beile-

I4 Das Thema wird von Thomas Prügl im Rahmen eines Wiener Forschungsprojekts sowie im Rahmen einer neuen Darstellung des Basler Konzils weiterhin bearbeitet.

I5 Vgl. so Dendorfer, Inszenierung (wie Anm.8), 5I.

I6 Vgl. Jürgen Miethke, Die Konzilien als Forum der öffentlichen Meinung im I5.Jahrhundert, in: Deutsches Archiv für Erforschung des Mittelalters 37, I98I, 736-773; Johannes Helmrath, Kommunikation auf den spätmittelalterlichen Konzilien, in: Hans Pohl (Hrsg.), Die Bedeutung der Kommunikation für Wirtschaft und Gesellschaft. (Vierteljahrschrift für Sozial- und Wirtschaftsgeschichte, Beih. 87.) Stuttgart I 989, I I6-I 72; Jürgen Miethke, Die Konzilien im I5. Jahrhundert als Drehscheiben internationaler Beziehungen, in: Konrad Krimm/Rainer Brüning (Hrsg.), Zwischen Habsburg und Burgund. Der Oberrhein als europäische Landschaft im I5.Jahrhundert. (Oberrheinische Studien, Bd. 2I.) Stuttgart 2003, 257-274; Johannes Helmrath, Diffusion des Humanismus und Antike-Rezeption auf den Konzilien von Konstanz, Basel und Ferrara-Florenz, in: Ludger Grenzmann/Klaus Grubmüller/Fidel Rädle/Martin Staehelin (Hrsg.), Die Präsenz der Antike im Übergang vom Mittelalter zur Frühen Neuzeit. Göttingen 2004, 9-54; Jürgen Miethke, Die großen Konzilien des I 5. Jahrhunderts als Medienereignis. Kommunikation und intellektueller Fortschritt auf den Großtagungen, in: Laurent Cesalli/Nadja Germann/Maarten J. F. M. Hoenen (Eds.), University, Council, City. Intellectual Culture on the Rhine (I300-I550). Turnhout 2009, 29I-322. 
gung zu fragen, ohne bestimmte Akteure, Praktiken oder Instanzen anhand moderner Vorstellungen von Staatlichkeit, Recht oder Legitimität zu privilegieren. Auch für die Erforschung synodaler Konfliktbearbeitung ermöglicht dies, historische Veränderungen der Entscheidungsfindung in einem akteursorientierten Zugriff zu rekonstruieren und unterschiedlich geartete Etappen und Episoden synodaler Entscheidungsfindung im Zusammenhang zu sehen.

Für die Erforschung synodaler Konfliktpraxis erscheint letztere Überlegung hochgradig relevant: Wir haben es angesichts des weitgehenden Fehlens eingespielter, geregelter und fest institutionalisierter Verfahren der politischen Konfliktlösung sowie zeitnaher Informationsübertragung durch Massenmedien im Mittelalter oft mit episodischen Verläufen der Konfliktbearbeitung und-lösung zu tun, die sich über längere Zeiträume hinziehen und eine Vielzahl räumlich-personaler Konstellationen einbeziehen konnten. Es liegt daher nahe, nach den jeweils historisch geltenden Regeln und Konventionen der Konfliktführung sowie nach Formen der Öffentlichkeit zu fragen, die insbesondere für das Früh- und Hochmittelalter intensiv erforscht worden sind. ${ }^{17}$

In der Untersuchung unterschiedlicher Konfliktstrategien stellt sich jedoch als Problem, dass trotz des relativ offenen Begriffs der Konfliktlösung moderne Konzepte wie ,Recht‘ und ,Politik‘ bzw. ,Verwaltung، erheblichen Einfluss gewinnen, sobald man nach diachroner Veränderung fragt. Zumindest seit dem I2.Jahrhundert ist etwa ein deutlicher Schub der Verrechtlichung synodaler Konfliktführung zu erkennen, der auf breitere gesellschaftliche Entwicklungen zurückzuführen ist. Die Verwissenschaftlichung der Theologie wirkt ebenfalls stark auf die Diskussionskultur der Zeit. Doch können die teilweise ja äußerst flexiblen Konfliktstrategien vormoderner Synoden nicht ohne Weiteres feststehenden Typen ,rechtlichen', , wissenschaftlichen' oder, politischen' Handelns zugeschlagen werden, ohne eine teleologische Sichtweise zu befördern.

Bei der Suche nach einem trennschärferen Analyseinstrumentarium für komplexe Konfliktbearbeitungsstrategien kann daher die Erforschung von Verfahren und

I7 Vgl. Claudia Garnier/Hermann Kamp (Hrsg.), Spielregeln der Mächtigen. Mittelalterliche Politik zwischen Gewohnheit und Konvention. Darmstadt 2010, sowie zur Erforschung politischer Öffentlichkeit Gerd Althoff, Formen und Funktionen öffentlicher Kommunikation im Mittelalter. (Vorträge und Forschungen, Bd. 5I.) Stuttgart 200I; Martin Kintzinger/Bernd Schneidmüller (Hrsg.), Politische Öffentlichkeit im Spätmittelalter. (Vorträge und Forschungen, Bd. 75.) Ostfildern 20 I I. 
Entscheidungen Anregungen geben, in der in letzter Zeit vor allem für die Frühe Neuzeit klare Ansätze und Operationalisierungen erarbeitet wurden. ${ }^{18}$ Als Idealtyp gesehen, zeichnet sich ein Verfahren durch eine feste, eventuell sogar schriftlich geregelte Form und einen zusammenhängenden Verfahrensablauf aus, der ein prinzipiell ergebnisoffenes Entscheiden vorsieht. ${ }^{\text {I9 }}$ Die Beteiligten eines ,Verfahrens' müssen dessen Ablauf im Vorhinein akzeptieren und nehmen im Rahmen des Verfahrens dann Rollen ein, die typischerweise von ihren sonstigen gesellschaftlichen Rollen geschieden sind. Die Legitimität der Entscheidung beruht dann nicht auf einer wie auch immer gearteten Richtigkeit des Ergebnisses, sondern wird als Ergebnis des Verfahrens erzeugt, das verbindliche Entscheidungen nicht nur herstellt, sondern gleichzeitig auch als solche darstellt und somit unmittelbare Bindewirkung erzeugt. ${ }^{20}$ Eine Vielzahl vormoderner synodaler Konfliktstrategien entspricht freilich nicht dem idealtypischen Schema eines derart ,autonomen' Verfahrens, sondern vielmehr dem der ,Verhandlung ${ }^{\text {'I }}$, bei der sich die Beteiligten nicht im Vorhinein auf eine Akzeptanz des Verfahrensergebnisses festlegen ließen, sondern sich die Handlungsalternativen des Protestes, des Hinausschiebens und des Verhandlungsabbruchs so lange offenhielten, bis das Ergebnis akzeptabel erschien.

Will man die Verfestigung bestimmter Typen der Konfliktlösung zu einer Verfahrensform untersuchen, muss es also darauf ankommen, die Grenzziehung zwischen formalisierten Verfahren und flexibleren Konfliktstrategien im Detail zu untersuchen. Wie Michael Sikora im Einzelnen dargestellt hat, kann man dazu etwa verschiedene Bestandteile verfahrensförmigen Vorgehens weiter isolieren, um dann zeitgenössische Diskussionen über einzelne Verfahrensfragen auszuwerten, wie sie gerade auf Synoden häufig vorkamen. Wie an verschiedenen historischen Konstellationen sichtbar wird, gerieten unter bestimmten Umständen auf Synoden

I8 Vgl. besonders Stollberg-Rilinger(Hrsg.), Vormoderne politische Verfahren (wie Anm. 7); dies./Krischer (Hrsg.), Herstellung und Darstellung von Entscheidungen (wie Anm. 7).

I9 Vgl. zur Definition Barbara Stollberg-Rilinger, Einleitung, in: dies./Krischer (Hrsg.), Herstellung und Darstellung von Entscheidungen (wie Anm. 7), 9-3I, bes. 9; dies., Einleitung, in: dies. (Hrsg.), Vormoderne politische Verfahren (wie Anm. 7), sowie Sikora, Der Sinn des Verfahrens (wie Anm. 7).

20 Vgl. Stollberg-Rilinger, Einleitung, in: dies./Krischer (Hrsg.), Herstellung und Darstellung von Entscheidungen (wie Anm.7), I I.

2 I So der Befund von Stollberg-Rilinger/Krischer (Hrsg.), Herstellung und Darstellung von Entscheidungen (wie Anm. 7), etwa Stollberg-Rilinger, Einleitung, 27. Vgl. auch Matthias Köhler, Verhandlungen, Verfahren und Verstrickung auf dem Kongress von Nimwegen I676-r679, in: Stollberg-Rilinger/Krischer (Hrsg.), Herstellung und Darstellung von Entscheidungen (wie Anm. 7), 4I I-44I, bes. 4I 3 f. 
unterschiedliche Facetten solcher Verfahrens- oder Verhandlungslösungen in den Fokus der Aufmerksamkeit. Während bei rechtlich akzentuierten Verfahren und Abstimmungen über Rolle, Zahl und Stimmrecht der einzelnen Beteiligten entschieden werden musste, war es bei theologisch ausgerichteten Disputationen notwendig, sich auf die zulässigen Argumente und Autoritäten zu einigen.

\section{Perspektiven und Akzente des vorliegenden Bandes}

Die Beiträge des vorliegenden Bandes zeigen immer wieder Querverbindungen zwischen diesen Fragekomplexen auf. Der einleitende und grundlegende Beitrag Thomas Graumanns zu Konfliktlösung auf Konzilien der Alten Kirche macht etwa deutlich, dass das Format der Synode seit der Antike für verschiedene Formen der Entscheidungsfindung prinzipiell offen war - vor allem für die rechtsförmige Konfliktlösung, die dann zumeist als gerichtliche Prüfung in Anlehnung an antike Gerichtsverfahren mit anschließender Disziplinierung einzelner Kontrahenten erschien, aber auch für die diskursive, unter Heranziehung von Autoritätentexten geführte Klärung durch Debatte. Wie Graumann herausstreicht, blieben diese Vorgehensweisen jedoch unscharf gegeneinander abgegrenzt. Kontrahenten, die zu einer Disputation angereist waren, konnten sich bei laufender Verhandlung plötzlich sozusagen vor Gericht wiederfinden. Besonders in der schriftlichen Publikation konnte das Zustandekommen von Beschlüssen angepasst dargestellt werden, auch wenn verschiedene Berichte unterschiedliche Erwartungen teils gegeneinander ausspielten. So verankerten schon die wichtigen spätantiken Synoden verschiedene Formen rechtlichen, argumentativ-deliberativen oder anderen Vorgehens im Erwartungshorizont.

Christoph Dartmanns Beitrag zu den Synoden des Westgotenreichs stellt noch deutlicher in den Mittelpunkt, wie problematisch die Überlieferung synodaler Beschlüsse sein konnte. An zwei unterschiedlich dokumentierten Konflikten arbeitet er heraus, welch intensive Vorläufe der Konfliktbearbeitung ans Tageslicht kommen, wenn nicht nur die synodale Versammlung selbst, sondern auch deren Vorlauf - oder, wie im Rahmen der Tagung mehrfach formuliert wurde, ihr ,Umfeld“ - in die Untersuchung einbezogen wird. Wie Dartmann deutlich zeigt, dürfen die westgotischen Synoden zumeist nicht als Orte der Entscheidungsfindung verstanden werden, sondern hatten vor allem die Funktion öffentlicher Publikation und Verbind- 
lichkeitsstiftung von Konfliktlösungen, die bereits vorher politisch ausgehandelt worden waren.

Der Beitrag Ernst-Dieter Hehls zu ,streitenden und einträchtigen Bischöfen“ im ottonischen und salischen Reich entwickelt eine aufschlussreiche Perspektive auf gruppenspezifisch und regional unterschiedliche Ausprägungen von Konfliktstrategien. Wie er an zahlreichen Konflikten zwischen Bischöfen, Päpsten und Herrschern herausarbeitet, darf man den Reichsbischöfen des Io. und I I.Jahrhunderts ein ausgeprägtes Streben nach konsensualer Einigung und einen intensiven Gebrauch vertraulicher und informeller Verhandlungsformen attestieren. Dies lag nicht zuletzt in religiösen Konsensidealen und in der Rücksichtnahme auf die Gemeinsamkeit mit dem Gegner im bischöflichen Amt begründet. Gleichzeitig macht Hehls Beitrag nachdrücklich deutlich, dass im Umfeld päpstlicher synodaler Konfliktlösung in Italien gleichzeitig ganz andere Formen der Entscheidungsfindung gepflegt wurden. Diese nahmen viel stärker rechtsförmigen Charakter an und waren auf den Papst hin organisiert. Diese Beobachtung legt den Zusammenhang zwischen regional dominierenden Mächtekonstellationen und der Bildung lokaler bzw. regionaler Konventionen der Konfliktaustragung sehr deutlich offen.

Der Beitrag von Constant J. Mews und Clare Monagle fokussiert im Gegensatz die graduelle Ausbildung neuer Konfliktstrategien über einen längeren Zeitraum. Am Beispiel synodaler Prozesse gegen frühscholastische Theologen von Berengar von Tours († I088) bis Gilbert von Poitiers († I I54) verfolgen die Autoren, wie schwierig und gewöhnungsbedürftig die textorientierte Diskussion bestimmter theologischer Positionen sein musste, solange es noch keine überregional verbindlichen Regeln und Methoden wissenschaftlicher Disputation gab. Wie Mews und Monagle zeigen, vollzieht sich im Übergang zu theologisch-diskursiver Konfliktführung nicht zuletzt eine Umschichtung von Autoritätsvorstellungen: Sie wurden nun nicht nur stärker schriftlich fixiert, sondern auch an bestimmte textuell-begriffliche Lehrsysteme gebunden. Um zu beschreiben, wie hier überregional verbindliche Normensysteme entstanden, könnte etwa der theologisch wie juristisch eingeführte Begriff eines ,Dogmatisierungsprozesses“ verwendet werden. ${ }^{22}$

Der Beitrag von Sita Steckel nutzt das Beispiel der Auseinandersetzungen zwi-

22 Vgl. zum Konzept der Dogmatisierung Georg Essen/Nils Jansen (Hrsg.), Dogmatisierungsprozesse in Recht und Religion. Tübingen 20 I I; Nils Jansen, Methoden, Institutionen, Texte, in: Zeitschrift der SavignyStiftung für Rechtsgeschichte, Germanistische Abteilung I28, 20I I, I-7 I. 
schen Papsttum und einer Gruppe französischer Bischöfe um die Privilegien der Bettelorden im späten I3.Jahrhundert, um die Öffentlichkeit synodaler Entscheidungen zu thematisieren. Das Beispiel des vertrackten Streits zwischen Papst und Prälaten, in dem nicht zuletzt um die Gültigkeit und Auslegung des Konzilsbeschlusses Omnis utriusque sexus von I 2 I 5 gerungen wurde, zeigt eine graduelle, durch die Konflikteskalation angeregte Qualitätsveränderung der von den Konflikparteien anvisierten Öffentlichkeit. Die reiche Parallelüberlieferung erlaubt in diesem Fall, den Hintergrund der verschiedenen, allmählich verknüpften Kommunikationsstrukturen genauer zu beschreiben.

Der Beitrag Melanie Brunners parallelisiert dagegen ganz bewusst die Verhandlungen über das franziskanische Armutsideal auf der Synode von Vienne I3 I I/I 2 mit der gleichzeitigen Konfliktlösungspraxis der päpstlichen Kurie. Wie Brunners instruktive Diskussion typischer Formen der Entscheidungsfindung durch Konsultationen und schriftliche Stellungnahmen am Papsthof demonstriert, hatte die $\mathrm{Ku}$ rie bereits ein dichtes Arsenal von Verfahren und Beratungstechniken entwickelt: Sie erzeugte durch semi-öffentliche Konsultationen zunächst eine hohe Mitwirkung aller Beteiligten, behielt die Entscheidung aber doch dem Papst vor. Das Konzil von Vienne beschreibt Brunner daher - in interessantem Gegensatz zu den Befunden Dartmanns für das Westgotenreich - als Ort der Öffentlichkeit und Diskussion, nicht jedoch der eigentlichen Beschlussfassung, die zumeist nachgelagert erfolgte.

Der Beitrag Volker Leppins stellt idealtypisch die ,diskursive“ und ,determinative‘ Entscheidungsfindung auf Konzilien gegeneinander, was insbesondere die Beobachtungen Graumanns zu Recht und Theologie weiterführt. Leppin diskutiert jedoch, was geschah, als im Zuge der frühen Reformation die rechtlich-determinative Institution der römischen Kirche zunächst ausgeschaltet wurde, und Entscheidungen somit deutlicher in deliberativer Form - oder, wie er nachzeichnet, durch das persönliche Charisma Luthers - abgestützt werden mussten. Wie Leppin argumentiert, musste die Schwächung der rechtlich-determinierenden Instanzen in einer Stärkung der diskursiven Entscheidungsfindung im Protestantismus resultieren.

Dies bildet einen interessanten Kontrapunkt zum Beitrag Andreas Pietschs, der am Beispiel der Synode von Dordrecht I6I8/ig das Ringen der Niederlande und weiterer reformierter Mächte um eine einheitliche Lehrentwicklung thematisiert. Wie er herausarbeitet, bewirkten die Interessengegensätze sowie die hinter der Synode deutlich hervortretende politische Konfliktsituation, dass die ursprünglich über Disputationen gesuchte Konfliktlösung schließlich zu einer synodalen Aburteilung 
der unterlegenen Partei geriet. Wie seine Diskussion der medialen Beobachtung der Synode zeigt, konnte die Verbindlichkeit der Entscheidung allerdings nur begrenzt sein, solange konkurrierende Berichterstattungen die Synode teils als Quell der Einheit, teils aber auch als Unrechtsversammlung darstellten. Wie in den von Graumann für die Spätantike geschilderten Fällen konnten also auch hier gegensätzliche Erwartungen an die Form der Konfliktführung gegeneinander ausgespielt werden. Die ungleich besseren kommunikationsgeschichtlichen Bedingungen der Frühen Neuzeit scheinen den Stimmen der Verlierer jedoch nun eine viel stärkere Resonanz in den Quellen zu bescheren.

\section{Resümierende Überlegungen}

Eine Zusammenschau der Beiträge zeigt vielerlei Querverbindungen auf und lässt bestimmte mittel- und langfristige Transformationen aufscheinen. Zu vier Aspekten - Öffentlichkeit, Verfahrensformen, historischen Verlaufsschemata und der Rolle von Religion - können abschließend einige Beobachtungen für zukünftige Forschungen festgehalten werden.

Eine erste Beobachtung betrifft die Stellung von Synoden im Rahmen breiterer politischer Willensbildungsprozesse und Öffentlichkeiten. Dass Synoden oftmals Orte der Konstitution von Öffentlichkeit waren und im Spätmittelalter einen Rahmen für weiter ausgreifende Meinungsbildungsprozesse bildeten, ist bekannt. ${ }^{23}$ Wie die Beiträge des Bandes in ihrer Gesamtheit zeigen, erweist sich die kommunikative Einbettung und mediale Vermittlung von Synoden jedoch als außerordentlich wichtige Achse mittel- und langfristiger Veränderungen.

Wie die Beiträge von Thomas Graumann, Christoph Dartmann und Ernst-Dieter Hehl zeigen, konnte eine synodale Versammlung in Antike, Früh- und Hochmittelalter in ganz unterschiedlichen Akzentuierungen entweder zentrales Forum kontroverser Debatten oder aber Instanz der Publikation und Verbindlichkeitsstiftung bereits gefundener Lösungen sein. Nicht unwesentlich erscheinen dabei die Kommunikationsnetzwerke und die Rhythmen der politischen Willensbildung, die synodale Treffen einrahmten. Einige der hier untersuchten Synoden ermöglichten erst

23 Vgl. oben bei Anm. I6. 
das Zusammentreffen ansonsten verstreuter Akteure, die dann im unmittelbaren Umfeld des face-to-face-Treffens Konfliktlösungen aushandelten. Waren jedoch von vornherein feste Kommunikationsnetzwerke etabliert, konnte der liturgische Rahmen der Synode verstärkt genutzt werden, um bestimmte Beschlüsse oder Inszenierungen herauszuheben.

Die hier diskutierten Befunde deuten jedoch nicht auf eine lineare Entwicklungsgeschichte vom Früh- zum Hochmittelalter oder darüber hinaus hin. Sie befördern eher die Vermutung, dass sich unter politisch günstigen Umständen bestimmte Formen der Nutzung von Synoden sozusagen ,einspielen‘ konnten und regional begrenzte Regimes typischer Konventionen hervorbrachten. Deutlich wird dies etwa an den von Hehl diskutierten Konfliktstrategien der Reichsbischöfe, die sich auf der Basis religiöser Konsensvorstellungen und hoher Achtung des bischöflichen Amtes entwickelten (was übrigens zur Erklärung der Entstehung ,konsensualer Herrschaft' beiträgt ${ }^{24}$ ). Wie Hehls kontrastierender Blick auf abweichende Konfliktlösungen in der Umgebung des Papsttums verdeutlicht, war die Konfliktkultur der Reichsbischöfe jedoch an den Rahmen der Reichskirche gebunden. Dartmanns Untersuchung der westgotischen Synoden zeigt einen parallelen Fall der Entwicklung typischer Strategien in einem geopolitisch begrenzten Umfeld - in seinem Beispiel tritt die Funktion von Synoden als Instanzen der Öffentlich- und Verbindlichmachung deutlich hervor.

Nicht erst im I5.Jahrhundert, sondern bereits seit dem Hochmittelalter zeigen sich größere Synoden und Konzilien jedoch auch als Resonanzräume und Arenen kontroverser Debatten. Schon im Vorfeld von Synoden wurden teils breitere, über die unmittelbaren Konfliktbeteiligten hinausgehende Öffentlichkeiten mobilisiert, um Druck aufzubauen und eine Konfliktlösung im eigenen Sinne zu erzwingen. Wie der Beitrag Melanie Brunners zeigt, konnten im Rahmen der synodalen Öffentlichkeit bald auch Alternativen der Konfliktlösung ausgelotet werden, ohne dass Entscheidungen getroffen oder publiziert wurden. Seit der Zeit der großen Konzilien zeichnet sich schließlich ab, dass konkreten synodalen Zusammenkünften eine lediglich rahmende Wirkung für weit ausgreifende, medial vermittelte Debatten zu-

24 Vgl. dazu Althoff, Spielregeln (wie Anm.6); Bernd Schneidmüller, Konsensuale Herrschaft. Ein Essay über Formen und Konzepte politischer Ordnung im Mittelalter, in: Paul-Joachim Heinig/Sigrid Jahns/ Hans-Joachim Schmidt/Rainer Christoph (Hrsg.), Reich, Regionen und Europa in Mittelalter und Neuzeit. Festschrift für Peter Moraw. (Historische Forschungen, Bd. 67.) Berlin 2000, 53-87. 
kommen konnte. War eine Mehrzahl politischer und kirchlicher Machtbereiche bereits gegeneinander abgegrenzt, wie es etwa im Falle der Synode von Dordrecht der Fall war, spiegelte die mediale Verarbeitung einer Synode dann teils schlicht diese Zersplitterung wieder.

Langfristige Entwicklungen, so eine zweite Beobachtung, können dabei mit dem Kontext unterliegender gesamtgesellschaftlicher Entwicklungen in Verbindung gebracht werden. Ganz wesentlich erweisen sie sich bei genauerem Hinsehen jedoch als kurz- und mittelfristige konflikt- und entscheidungstheoretische Lernprozesse. Dies zeigt sich im Rahmen des vorliegenden Bandes insbesondere für den Wandel von Formen der Entscheidungsfindung. Der von Graumann hervorgehobene, „im Kern hybride Charakter“ 25 von Synoden bewirkte zunächst eine große Offenheit und Flexibilität verschiedener Modi und Herangehensweisen. Der Beobachtung synodaler Konfliktlösung durch verschiedene Öffentlichkeiten kam aber offenbar eine katalytische Funktion zu: Teilnehmer und Beobachter hatten bestimmte Erwartungen, die an Synoden und synodale Konfliktlösung gerichtet wurden. Wollte man eine spezifische Gruppe mobilisieren, so galt es diese möglichst gezielt anzusprechen und die vorgeschlagene Konfliktlösung gegenüber ihren spezifischen Erwartungen zu rechtfertigen.

Eine Habitualisierung bestimmter Verfahren etablierte sich dann typischerweise unter bestimmten Bedingungen: Wo Herrscher, Päpste oder auch besonders einträchtige Bischöfe genügend Macht auf sich versammeln konnten und im Besitz von Druck- und Sanktionsmitteln waren, konnten Verfahrenslösungen von einer überlegenen Partei schlicht durchgesetzt werden. ${ }^{26}$ War dies nicht der Fall, so scheinen Routinisierungen und Wiederholungen bestimmter, bereits bekannter Formen als wichtiger Hintergrund der Formalisierungen bestimmter Vorgehensweisen auf. Die zeitweise fehlende Akzeptanz für festgelegte, im Vorhinein strukturierte Verfahren erklärt sich dagegen offenbar nicht aus einem Fehlen rechtlicher oder rationaler ,Mentalität‘ in der Vormoderne bzw. im frühen und hohen Mittelalter gegenüber der Zeit ab dem I2.Jahrhundert. Im Gegenteil - die Akzeptanz verfahrensförmiger

\footnotetext{
25 Vgl. unten 4I.

$26 \mathrm{Zu}$ beachten wären insbesondere Vorgänge der Transformation von Konfliktstrategien aufgrund einer Nachfrage ,von unten', wie sie etwa für Justiznutzung diskutiert worden sind, vgl. Daniel L. Smail, The Consumption of Justice. Emotions, Publicity, and Legal Culture in Marseille, I264-I423. Ithaca 2003; sowie allgemein Willem Pieter Blockmans/André Holenstein/Jon Mathieu (Eds.), Empowering Interactions. Political Cultures and the Emergence of the State in Europe, I300-I900. Farnham 2009.
} 
Konfliktlösung dürfte zu wesentlichen Teilen auf der Bekanntheit und Absehbarkeit von Verfahrensformen beruhen. Diese Absehbarkeit geriet in Zweifel, wo unterliegende Veränderungen im Verständnis des Rechts oder der doktrinalen Wahrheitsfindung den Ablauf synodaler Debatten zu einer unsicheren Angelegenheit werden ließen.

In den schwierigen Theologenprozessen des Hochmittelalters, die von Mews und Monagle diskutiert werden, bestand etwa schon aufgrund der intensiven wissensgeschichtlichen Transformationsprozesse der Zeit ${ }^{27}$ zunächst keinerlei Klarheit über die maßgeblichen Begrifflichkeiten und Methoden, was wiederholt zum Streit über Verfahrensformen führte. Als durch die enge Verbindung von Papstkirche und Universitäten im I 3. und I4.Jahrhundert jedoch ein relativ stabiles, berechenbares Normengerüst bereitstand ${ }^{28}$, konnte, wie Brunner zeigt, sogar ein allgemeines Konzil wie das von Vienne I3I I/I 2 zum Forum der öffentlichen Äußerung kontroverser Argumente werden. Auch die Einholung von Gutachten im Rahmen kurialer Konsultationen hatte zu diesem Zeitpunkt längst Bezüge zu eingespielten Formen der Argumentation und folgte einem erwartbaren Verlauf. In anderen historischen Situationen griff man zu situativen Klärungen, welche Verfahrensform gewählt oder welche Text- und Autoritätenbasis der Diskussion zugrunde liegen sollte - so einerseits auf den wegweisenden Konzilien der Spätantike, andererseits unter den stark veränderten Bedingungen der nachreformatorischen Zeit.

Ein Abgleich gesellschaftlicher Kontexte und spezifischer Debatten um die Form synodaler Konfliktlösung macht somit auch das fortgesetzte Nebeneinander einer auf Ehrwahrung orientierten und doch immer stärker verrechtlichten Gesellschaft verständlich: Waren bestimmte Rollen und Vorgehensweisen des Streitens fest eingespielt und gar durch entsprechende Formalisierung gestützt, wie etwa die scholastischen Disputationsformen, wurde es auch möglich, öffentlich ohne Verlust der Ehre kontroverse Positionen zu vertreten. Als wesentlich erweisen sich weniger die

27 Vgl. die Untersuchung der von Mews und Monagle behandelten Fälle aus wissensgeschichtlicher Sicht bei Sita Steckel, Kulturen des Lehrens im Früh- und Hochmittelalter. Autorität, Wissenskonzepte und Netzwerke von Gelehrten. (Norm und Struktur, Bd. 39.) Köln/Weimar/Wien 20I I, 886-924, I085-I I 77. 28 Vgl. zur Verfestigung von Verfahrensformen Jürgen Miethke, Theologenprozesse in der ersten Phase ihrer institutionellen Ausbildung. Die Verfahren gegen Peter Abaelard und Gilbert von Poitiers, in: Viator 6, I975, 87-II9; ders., Papst, Ortsbischof und Universität in den Pariser Theologenprozessen des I3.Jahrhunderts, in: Albert Zimmermann (Hrsg.), Die Auseinandersetzungen an der Pariser Universität im XIII.Jahrhundert. (Miscellanea Mediaevalia, Bd. ro.) Berlin/New York I 976, 52-94. 
Signaturen ganzer Epochen oder Teilepochen als vielmehr Unterschiede zwischen geregeltem, erwartbaren und damit situativ einschätzbarem Streiten und spontan und ungeregelt ausbrechendem Konflikt, der in verschiedenen ,Streitkulturen“ bis einschließlich denen der Gegenwart weiterhin als Ordnungsstörung gilt. ${ }^{29}$

Ein weiterer Komplex von Überlegungen betrifft Annahmen zu langfristigen Verläufen und kann schließlich zur eingangs gestellten Frage nach der Besonderheit synodaler Konfliktführung zurückführen. In den letzten Jahren sind nicht nur einzelne historische Verlaufsannahmen, sondern auch die ihnen zugrunde liegenden theoretischen Modelle und großen Narrative in die Kritik geraten. Stark diskutiert sind nicht zuletzt die großen Erzählungen, die das gegenseitige Verhältnis von Religion und Politik betreffen - beziehungsweise die, erweitert formuliert, das Verhältnis gesellschaftlicher Sphären wie Religion, Politik, Recht, Wirtschaft historisieren und dabei nach dem historischen Verlauf von größeren Differenzierungsprozessen fragen. ${ }^{30}$ Die Untersuchung von Synoden, die gleichermaßen politische wie religiöse Versammlungen waren und sowohl zu rechtlichen wie gelehrt-theologischen Formen der Wahrheitsfindung greifen konnten, erweist sich für diese Frage als außerordentlich aufschlussreich: Gerade auf Synoden legten antike, mittelalterliche oder frühneuzeitliche Akteure eine Vielzahl verschiedener Abgrenzungen dieser Sphä-

29 Verschiedene Ansätze zu ,Streitkulturen“ u.a. in: Uwe Baumann/Arnold Becker/Astrid Steiner-Weber (Hrsg.), Streitkultur. Okzidentale Traditionen des Streitens in Literatur, Geschichte und Kunst. (Super alta perennis, Bd. 2.) Göttingen 2008; Magnus Eriksson/Barbara Krug-Richter (Hrsg.), Streitkulturen. Gewalt, Konflikt und Kommunikation in der ländlichen Gesellschaft (I6.-I9.Jahrhundert). Köln/Weimar/Wien 2003; Gunther Gebhard/Oliver Geisler/Steffen Schröter (Hrsg.), StreitKulturen. Polemische und antagonistische Konstellationen in Geschichte und Gegenwart. Bielefeld 2008.

30 Die Debatte hat sich von einer Kritik großer Erzählungen in Richtung der historischen Operationalisierung von Differenzierungstheorien sowie in Richtung neuer Periodisierungen entwickelt. Vgl. etwa die verschiedenen kritischen Ansätze bei Ulrich Willems/Detlef Pollack/Helene Basu/Thomas Gutmann/Ulrike Spohn (Hrsg.), Moderne und Religion. Kontroversen um Modernität und Säkularisierung. Bielefeld 20I 2; Karl Gabriel/Christel Gärtner/Detlef Pollack(Hrsg.), Umstrittene Säkularisierung. Soziologische und historische Analysen zur Differenzierung von Religion und Politik. 2. Aufl. Berlin 2014; Alexandra Walsham, The Reformation and the „Disenchantment of the World“ Reassessed, in: Historical Journal 5I, 2008, 497-528; Ansätze zur historischen Erforschung von Differenzierung in Ludger Körntgen/Dominik Waßenhoven (Hrsg.), Religion and Politics in the Middle Ages. Germany and England by Comparison/Religion und Politik im Mittelalter. Deutschland und England im Vergleich. Berlin/New York 20r3; Detlef Pollack/Sita Steckel/Gerd Althoff, Differenzierung von Religion und Politik im Mittelalter, in: Frühmittelalterliche Studien 47, 2013, 273-377; zur Periodisierung vgl. auch Alexandra Walsham, Migrations of the Holy. Explaining Religious Change in Medieval and Early Modern Europe, in: Journal of Medieval and Early Modern Studies 44, 20I4, 24I-280. 
ren vor. Angesichts von politisierten Konflikten tadelten sie häufig die Vermischung von Logiken, die ihrer Ansicht nach getrennt bleiben sollten - etwa Konfliktentscheide, hinter denen wirtschaftliche Motive vermutet wurden, oder politisch opportune Eingriffe in Wahrheitskonflikte. Gerade für Synoden sind solche abgrenzenden Unterscheidungen vielfach zu beobachten - etwa in den von Christoph Dartmann diskutierten Aufforderungen synodaler Ordines zur Vermeidung von Gewalt oder Bestechlichkeit. ${ }^{3 \mathrm{I}}$ Damit wurden implizit Grenzarbeiten zwischen sozialen Feldern wie Religion, Politik, Wissenschaft oder Recht vorgenommen und vorhandene gesellschaftliche Differenzierungen adaptiert (oder auch festgeschrieben). ${ }^{32}$ Ihre Offenheit für rechtliche, religiöse oder sonstige, im weiten Sinne politische Argumente machte Synoden somit zu Arenen der Weichenstellung für längerfristige gesellschaftliche Differenzierungsprozesse.

In jüngeren Forschungen ist bereits begonnen worden, diese Vorgänge genauer einzuordnen - doch ist man sich bislang nicht einig geworden, wie vormoderne Praktiken der Konfliktlösung mit größeren strukturellen Prozessen zu verknüpfen wären. Da traditionellerweise bestimmte Formen der Konfliktlösung als typisch für das Mittelalter oder die Neuzeit, die Vormoderne oder Moderne angesehen wurden, ist die enge und äußerst dynamische Verknüpfung verschiedener Formen und Dimensionen von Konflikten häufig als Indiz für das Fehlen gesellschaftlicher Differenzierung gewertet worden. So hat Gerd Althoff pointiert auf die „Unschärfe und Mehrdeutigkeit zentraler Grundsätze, Gewohnheiten und Regeln“ im Kontext der hochmittelalterlichen politischen Willensbildung hingewiesen. ${ }^{33}$ In anderem $\mathrm{Zu}$ sammenhang stellte er jedoch die Trennung gesellschaftlicher Sphären heraus. ${ }^{34}$ Ähnlich bringt Barbara Stollberg-Rilinger die hohe Tendenz vormoderner Akteure

\footnotetext{
3 I Vgl. unten 68.

32 Zur differenzierungstheoretischen Frage nach Grenzarbeiten an sozialen Feldern vgl. die Überlegungen von Astrid Reuter, Grenzarbeiten am religiösen Feld. Religionsrechtskonflikte und Kontroversen im Verfassungsstaat, in: Jamal Malik (Hrsg.), Religionsproduktivität in Europa. Markierungen im religiösen Feld. (Vorlesungen des interdisziplinären Forums Religion der Universität Erfurt, Bd. 6.) Münster 2009, IOI-I I6; dies., Religion in der verrechtlichten Gesellschaft. Rechtskonflikte und öffentliche Kontroversen um Religion als Grenzarbeiten am religiösen Feld. (Critical Studies in Religion, Vol. 5.) Göttingen 20 I4, 4359.

33 Vgl. Gerd Althoff, Libertas ecclesiae oder die Anfäge der Säkularisierung im Investiturstreit?, in: Gabriel/Gärtner/Pollack (Hrsg.), Umstrittene Säkularisierung (wie Anm. 30), 78-ıoo.

34 Vgl. Gerd Althoff, Differenzierung zwischen Kirche und Königtum im Mittelalter. Ein Kommentar zum Beitrag Detlef Pollacks, in: Frühmittelalterliche Studien 47, 2013, 353-367.
} 
zum Abbruch verfahrensförmiger Konfliktlösung mit unterschiedlichen Differenzierungszuständen in Vormoderne und Moderne in Verbindung. Sie weist gleichzeitig jedoch darauf hin, dass sich in bestimmten Bereichen auch in der Moderne keine klaren Verfahrensformen etablierten konnten. ${ }^{35}$

Aus der hier verfolgten Perspektive erscheint daher fraglich, ob der häufige Wechsel verschiedener Handlungslogiken auf vormodernen Synoden (oder die hohe Fluidität gesellschaftlicher Sphären allgemein) als Zeichen fehlender funktionaler Differenzierung vormoderner Gesellschaften und historisches Residuum zu werten ist. Man könnte auch umgekehrt formulieren, dass die Grenzen verschiedener sozialer Sphären oder Felder im Mittelalter offenbar in relativ dynamischen Wandlungsprozessen begriffen waren. Dann stünde man freilich vor einer etwas überraschenden Gegenüberstellung - die Vormoderne erschiene als Epoche besonders fluider, die Moderne aber als Zeit einer statischen, gleichbleibenden gesellschaftlichen Differenzierung.

Nuanciert man die dichotomische Gegenüberstellung von Vormoderne und Moderne, ließen sich dagegen überzeugendere Verlaufsannahmen postulieren: Wie bereits erwähnt, ist offenbar nicht von gleichbleibenden Signaturen ganzer (Teil-) Epochen, sondern von mittelfristigen Stabilisierungen und Routinisierungen in bestimmten Bereichen gesellschaftlicher Konfliktlösung auszugehen. Da geopolitische, mediale oder epistemologische Wandlungen etablierte Formen der Konfliktlösung immer wieder destabilisierten, wurden sie jedoch in größeren oder kleineren Episoden der Adaptation wiederholt neu verhandelt. Solche Episoden der Anpassung einschlägiger Konventionen und Ideale sind im Zusammenhang mit synodaler Konfliktlösung von der Spätantike bis in die Frühe Neuzeit immer wieder zu beobachten; sie setzen sich offensichtlich bis in die Gegenwart fort. Dass mit der Moderne tatsächlich in vielen Bereichen eine Stabilisierung der Konfliktlösung zu konstatieren ist, dürfte dagegen einerseits mit technischen und medialen Normierungsmöglichkeiten zusammenhängen, andererseits die zunehmend gefestigte Dominanz bestimmter (etwa juristischer und politischer) Eliten widerspiegeln.

Die Erforschung von Synoden bietet sich angesichts dieser Überlegung in besonderer Weise an, um historische Abgrenzungsprozesse bestimmter gesellschaftlicher Sphären oder Felder aufzuschlüsseln. Kirchliche Versammlungen erscheinen prin-

35 Vgl. Stollberg-Rilinger, Einleitung, in: dies./Krischer (Hrsg.), Herstellung und Darstellung von Entscheidungen (wie Anm. 7), i 2 f. 
zipiell von einer Verschränkung religiöser, rechtlicher, wissenschaftlicher und politischer Logiken charakterisiert. Sie erlauben daher, historische Dynamiken zwischen diesen Bereichen wie in einem Brennglas zu fokussieren. Anstatt sie mit einem linearen Verlaufsschema zu konfrontieren, liegt es nahe, bestimmte Fragen an synodale Konfliktführung zu richten - etwa zu analysieren, wo Veränderungen auf unterliegende mediale oder politische Wandlungen reagieren, oder welche Rolle bestimmten Eliten in der Adaptation etablierter Konfliktführungsmechanismen zukam.

Letztere Frage wird im vorliegenden Band wiederholt tangiert: Neben politischen Akteuren treten in den Beiträgen beispielsweise gelehrte Eliten hervor, die in der Lage waren, die Wissensbasis politischer, rechtlicher oder religiöser Entscheidungspraxis zu stabilisieren oder zu dynamisieren. Für die Konzilien des I 5.Jahrhunderts ist dies bereits mehrfach beobachtet worden - in einer Fallstudie demonstrierte etwa Thomas Prügl ${ }^{36}$, wie der ,konziliar‘ argumentierende Johannes von Ragusa I434 in Basel die biblische und patristische Überlieferung verstärkt heranzog, um gegen die stark kanonisch-rechtlich ausgearbeitete papalistische Position des Juan de Torquemada zu streiten. Johannes Helmrath wies auf das Phänomen der „Theologisierung“ auf den Konzilien des I 5.Jahrhunderts hin, da immer wieder theologische und biblische Argumente in Stellung gebracht und von juristischen Diskursen zudem auch aufgenommen wurden. ${ }^{37}$

Auch andere mittelfristige Tendenzen könnte man aber in Bezug zu wechselnden Konstellationen politischer und gelehrter Eliten stellen: Der Beitrag von Mews und Monagle in diesem Band deutet an, dass das entstehende, überregional verbindliche

36 Vgl. Thomas Prügl, Modelle konziliarer Kontroverstheologie Johannes von Ragusa und Johannes von Torquemada, in: Helmrath/Müller (Hrsg.), Die Konzilien von Pisa (wie Anm. I I), 257-287.

37 Vgl. die Bemerkungen von Helmrath, Das Basler Konzil (wie Anm. I I), 4I 7-420; Helmrath/Müller, Zur Einführung (wie Anm. I I), 20, und Thomas Prügl, Das Schriftargument zwischen Papstmonarchie und konziliarer Idee. Biblische Argumentationsmodelle im Basler Konziliarismus, in: Andreas Pečar/Kai Trampedach (Hrsg.), Die Bibel als politisches Argument. Voraussetzungen und Folgen biblizistischer Herrschaftslegitimation in der Vormoderne. (HZ, Beih. NF., Bd. 43.) München 2007, 2 I9-24I; Thomas Woelki, Theologische und juristische Argumente in den Konzilstraktaten des Lodovico Pontano († I439), in: Péter Erdö/ Szabolcs Anzelm Szuromi (Eds.), Proceedings of the Thirteenth International Congress of Medieval Canon Law. Esztergom, 3-8 August 2008. (Monumenta Iuris Canonici, Ser. C: Subsidia, Vol. I4.) Vatikanstadt 20Io, 747-763; Hermann Schüssler, Der Primat der Heiligen Schrift als theologisches und kanonistisches Problem im Spätmittelalter. (Veröffentlichungen des Instituts für europäische Geschichte, Abt. für Abendländische Religionsgeschichte, Bd. 86.) Wiesbaden I977. 
Normensystem des Reformpapsttums mit seiner oft verfahrensförmigen Konfliktlösung wesentlich auf einem Bündnis mit bestimmten Gelehrtengruppen beruhte, das in konfliktreichen Episoden erstritten wurde. ${ }^{38}$ Die Theologisierung auf den Reformkonzilien könnte man als Auseinanderbrechen dieses Bündnisses im Zuge des Schismas und der kirchlichen Krise sehen, dem bereits lokale Konflikte wie der von Steckel behandelte Bettelordensstreit vorausgegangen waren. Im Zuge der Konzilien wurden Gelehrte dann zwar oft zu Trägern einer vereinheitlichenden Bewegung zur Reform. Doch hatten sie in den stark verdichteten spätmittelalterlichen Bildungslandschaften längst lokale Zugehörigkeiten entwickelt und damit auch lokal geprägte kulturelle und religiöse Identitäten ausgebildet. Der Beitrag Volker Leppins verdeutlicht, wie in der Folge die gelehrte Entscheidungsfindung im frühen I6.Jahrhundert nunmehr für lokale reformatorische Bewegungen instrumentalisiert wurde. Das Legitimitätsdefizit von Lehrentscheidungen in reformatorischen Gemeinschaften wurde so zunächst durch deliberative Formen der Konfliktlösung wie die Disputation aufgefangen. Wie der Beitrag Andreas Pietschs zeigt, stand jedoch im Laufe der Frühen Neuzeit auch neuen Bündnissen zwischen gelehrten Akteuren und politischen Mächtekonstellationen nichts im Wege, so dass es zur Stabilisierung mehrerer neuer, nunmehr politisch/lokal und konfessionell geprägter Normensysteme kommen konnte, wie sie beispielsweise auf der reformierten Synode von Dordrecht grundgelegt wurden.

Eine letzte Beobachtung betrifft die Rolle von Religion in der Konfliktbearbeitung vormoderner Synoden und mutmaßlich auch in der Veränderung anderer historischer Kulturen des Entscheidens. Religion tritt in einem offenen, lineare Verlaufsschemata vermeidenden Zugriff als besonders interessanter Dynamisierungsfaktor hervor: In den beschriebenen Tendenzen der Verrechtlichung und der Formalisierung von Verfahren liegen offensichtlich klare Entwicklungen von religiösen zu rechtlichen, wissenschaftlichen oder politischen Formen der Konfliktlösung. Doch erweisen sich auch Bezüge auf religiöse Argumente und Entscheidungsmodi immer wieder als aktuell - ja treten geradezu verstärkt auf, wenn Umbrüche zu verzeichnen sind.

Die Entwicklung religiöser Logiken des Entscheidens ist also offenbar nicht auf einen reinen Bedeutungsrückgang zu reduzieren - auch wenn auffällt, dass be-

38 Vgl. dazu zuletzt Clare Monagle, Orthodoxy and Controversy in Twelfth-Century Religious Discourse. Peter Lombard's Sentences and the Development of Theology. (Europa Sacra, Vol.8.) Turnhout 2013. 
stimmte soziale Räume, darunter solche der Konfliktaustragung, teils immer stärker von religiösen Logiken freigehalten werden, was einem bestimmten Verständnis von Säkularisierung entspricht. ${ }^{39}$ Die Rolle religiöser Darstellung und Deutung von Konfliktstrategien ließe sich eher fassen, indem man ihre dynamisierende und Übergänge legitimierende Funktion hervorhebt: Immer wieder konnte auf die Notwendigkeit zur Wahrheitsfindung verwiesen werden, um politische Entscheidungen anzugreifen, konnte religiöse Eintracht über Rechtsformen und Interessen gestellt werden, konnte schließlich in Krisensituation das direkte Eingreifen Gottes herbeigewünscht oder als Legitimation aufgerufen werden werden. Gerade die Überwindung bestehender rechtlicher und politischer Zustände - paradigmatisch zusammengefasst im mittelalterlichen Ausspruch, „[Christus] non dixit: ego sum consuetudo" 40 - war den christlichen Traditionen schon aufgrund ihrer Ursprünge als Minderheitenposition im römischen Imperium tief eingeschrieben. Sehr häufig finden wir daher neue Formen synodaler Entscheidungsfindung religiös begründet - genauso häufig wie allerdings Proteste gegen synodale Entscheidungen in religiösen Formen geäußert wurden, was teils wiederum neue Formen der Entscheidungsfindung vorbereitete. An die Stelle linearer Säkularisierungsdynamiken dürfte im Falle der langfristigen Entwicklung von Synoden - wie sicherlich auch im Falle weiterer politischer Vergemeinschaftungen der Vormoderne - also eine Abfolge charismatischer Neubeginne mit jeweils darauffolgenden Institutionalisierungen nach dem Modell Max Webers treten. ${ }^{4 \mathrm{I}}$ Es bleibt zu hoffen, dass nicht nur einzelne Konfliktfälle auf vormodernen Synoden, sondern auch derartige große Linien weiteres Interesse der Forschung finden.

39 Vgl. etwa Alexandra Walsham, Zu Tisch mit Satansjüngern. Geistliche und weltliche Soziabilität im nachreformatorischen England, in: Andreas Pietsch/Barbara Stollberg-Rilinger (Hrsg.), Konfessionelle Ambiguität. Uneindeutigkeit und Verstellung als religiöse Praxis in der Frühen Neuzeit. (Schriften des Vereins für Religionsgeschichte, Bd. 2 I4.) Göttingen 2013, 285-3I3, sowie zum Verständnis von Säkularisierung Charles Taylor, A Secular Age, Cambridge, MA 2007, If.

40 Vgl. dazu André Gouron, Non Dixit. Ego Sum Consuetudo, in: Zeitschrift der Savigny-Stiftung für Rechtsgseschichte, Kanonistische Abteilung 74, I988, I33-I40.

4I Vgl. zu Anregungen Webers für die Mediävistik zuletzt David L. d'Avray, Medieval Religious Rationalities. A Weberian Analysis. Cambridge 20 o. 